\title{
Hidden charm and bottom molecular states
}

\author{
F.-K. Guo, ${ }^{1}$ C. Hidalgo-Duque, ${ }^{2}$ J. Nieves, ${ }^{2}$ A. Ozpineci,${ }^{3}$ and M. Pavón Valderrama ${ }^{4}$ \\ ${ }^{1}$ Helmholtz-Institut für Strahlen- und Kernphysik and Bethe Center \\ for Theoretical Physics, Universität Bonn, D-53115 Bonn, Germany \\ ${ }^{2}$ Instituto de Física Corpuscular (IFIC), Centro Mixto CSIC-Universidad de Valencia, \\ Institutos de Investigación de Paterna, Aptd. 22085, E-46071 Valencia, Spain \\ ${ }^{3}$ Middle East Technical University - Department of Physics TR-06531 Ankara, Turkey \\ ${ }^{4}$ Institut de Physique Nucléaire, Université Paris-Sud, IN2P3/CNRS, F-91406 Orsay Cedex, France
}

\begin{abstract}
We investigate heavy quark symmetries for heavy light meson-antimeson systems in a contactrange effective field theory. In the $\mathrm{SU}(3)$ light flavor limit, the leading order Lagrangian respecting heavy quark spin symmetry contains four independent counter-terms. Neglecting $1 / m_{Q}$ corrections, three of these low energy constants can be determined by theorizing a molecular description of the $X(3872)$ and $Z_{b}(10610)$ states. Thus, we can predict new hadronic molecules, in particular the isovector charmonium partners of the $Z_{b}(10610)$ and the $Z_{b}(10650)$ states. We also discuss hadron molecules composed of a heavy meson and a doubly-heavy baryon, which would be related to the heavy meson-antimeson molecules thanks to the heavy antiquark-diquark symmetry. Finally, we also study the $X(3872) \rightarrow D^{0} \bar{D}^{0} \pi^{0}$ decay, which is not only sensitive to the short distance part of the $X(3872)$ molecular wave function, as the $J / \psi \pi \pi$ and $J / \psi 3 \pi X(3872)$ decay modes are, but it is also affected by the long-distance structure of the resonance. Furthermore, this decay might provide some information on the interaction between the $D \bar{D}$ charm mesons.
\end{abstract}

PACS numbers: 03.65.Ge, 13.75.Lb, 14.40Pq, 14.40Rt

\section{HEAVY MESON MOLECULES}

The recent discoveries of exotic heavy quarkonium states revived old expectations on the possible existence of molecular (loosely bound mesonic colour singlets) states [1, 2]. The most likely candidates are the $X(3872)$ resonance [3] and the isovector $Z_{b}(10610)$ and $Z_{b}(10650)$ states $[4,5]$. The proximity of the $X(3872)$ to the $D \bar{D}^{* 0}$ threshold and its decay properties have led to the general acceptance that it is a weakly bound state, with quantum numbers $J^{P C}=1^{++}[\underline{6}]$, generated from $D^{0} \bar{D}^{* 0}, D^{+} \bar{D}^{*-}$ coupled channel interactions $[7]^{1}$. Heavy quark symmetries deduced from QCD provide an adequate framework to study these systems. Thus, heavy quark spin symmetry (HQSS) implies that molecular states should appear in HQSS multiplets, while from heavy flavor symmetry (HFS), similarities in the bottom and charm spectra might be expected. Indeed, combining both HQSS and HFS, various partners of the $X(3872)$ and the isovector $Z_{b}^{\prime s}$ states can be predicted [8 [15].

Actually, HQSS heavily constrains the low-energy interactions among heavy hadrons [9, 11, 13, 14, 16]. As long as the hadrons are not too tightly bound, they will not probe the specific details of the interaction binding them at short distances. Moreover, each of the constituent heavy hadrons will be unable to see the internal structure of the other heavy hadron. This separation of scales can be used to formulate an effective field theory (EFT) description of hadronic molecules [11, 13] compatible with the approximate nature of HQSS. At very low energies, the leading order (LO) interaction between pseudoscalar and vector charmed $\left(D^{0}, D^{+}, D^{* 0}, D^{*+}\right)$ and anti-charmed $\left(\bar{D}^{0}, D^{-}, \bar{D}^{* 0}, D^{*-}\right)$ mesons $^{2}$ can be described just in terms of a contact-range potential, which is constrained by HQSS [13 15]. Pion exchange and particle coupled-channel ${ }^{3}$ effects turn out to be sub-leading [13, 17].

The LO Lagrangian contains four independent terms in the $\mathrm{SU}(3)$ flavor limit [14], which strength is set by two isoscalar $C_{0 A}$ and $C_{0 B}$ and two isovector $C_{1 A}$ and $C_{1 B}$ low energy constants (LEC's). The (contact) interaction potential is used as kernel of a two body elastic Lippmann-Swinger equation (LSE). The LSE shows an ill-defined ultraviolet (UV) behaviour, and it requires a regularization and renormalization procedure (see Refs. 14, 15] for details). The LSE non-perturbative re-summation restores elastic unitarity and provides a non-analytical structure of the scattering amplitudes. Bound states $\left(D^{(*)} \bar{D}^{(*)}\right.$ or $B^{(*)} \bar{B}^{(*)}$ molecules) correspond to poles of the $T$-matrix below threshold on the real axis in the first Riemann sheet of the complex energy, while virtual states, that if located near threshold might be relevant, show up in the second Riemann sheet.

\footnotetext{
${ }^{1}$ When we refer to $D^{0} \bar{D}^{* 0}, D^{+} D^{*-}$, or in general $D \bar{D}^{*}$, we are actually referring to the combination of these states with their charge conjugate ones in order to form a state with well-defined C-parity.

2 The discussion runs in parallel for the bottom sector.

${ }^{3}$ We do not refer to charge channels, but rather to the mixing among the $D \bar{D}, D \bar{D}^{*}, D^{*} \bar{D}^{*}$ pairs in a given $I J C$ (isospin, spin and charge conjugation) sector.
} 
TABLE I: Heavy meson-heavy meson combinations having the same contact term as the $X(3872)$ and $Z_{b}(10610)$, and the predictions of the masses, which are understood to correspond to bound states except if we write "V" in parenthesis for denoting a virtual state. $\dagger$ : increasing the strength of the potential to account for the various uncertainties, the virtual pole evolves into a bound state. Masses are given (MeV units) for two UV regulators. For further details see [15].

\begin{tabular}{|c|c|c|c|c|}
\hline$I\left(J^{P C}\right)$ & States & $M(\Lambda=0.5 \mathrm{GeV})$ & $M(\Lambda=1 \mathrm{GeV})$ & Measurements \\
\hline $0\left(1^{++}\right)$ & $D \bar{D}^{*}$ & 3871.68 (input) & 3871.68 (input) & $3871.68 \pm 0.17[20]$ \\
\hline $0\left(2^{++}\right)$ & $D^{*} \bar{D}^{*}$ & $4012_{-5}^{+4}$ & $4012_{-12}^{+5}$ & $?$ \\
\hline $0\left(1^{++}\right)$ & $B \bar{B}^{*}$ & $10580_{-8}^{+9}$ & $10539_{-27}^{+25}$ & $?$ \\
\hline $0\left(2^{++}\right)$ & $B^{*} \bar{B}^{*}$ & $10626_{-9}^{+8}$ & $10584_{-27}^{+25}$ & $?$ \\
\hline $0\left(2^{+}\right)$ & $D^{*} B^{*}$ & $7322_{-7}^{+6}$ & $7308_{-20}^{+16}$ & $?$ \\
\hline $1\left(1^{+-}\right)$ & $B \bar{B}^{*}$ & $10602.4 \pm 2.0$ (input) & $10602.4 \pm 2.0$ (input) & $\begin{array}{c}10607.2 \pm 2.0[4] \\
10597 \pm 9[21]\end{array}$ \\
\hline $1\left(1^{+-}\right)$ & $B^{*} \bar{B}^{*}$ & $10648.1 \pm 2.1$ & $10648.1_{-2.5}^{+2.1}$ & $\begin{array}{c}10652.2 \pm 1.5[4] \\
10649 \pm 12[21]\end{array}$ \\
\hline $1\left(1^{+-}\right)$ & $D \bar{D}^{*}$ & $3871_{-12}^{+4}(\mathrm{~V})$ & $3837_{-35}^{+17}(\mathrm{~V})$ & $\begin{array}{l}3899.0 \pm 3.6 \pm 4.9[22] \\
3894.5 \pm 6.6 \pm 4.5[23]\end{array}$ \\
\hline $1\left(1^{+-}\right)$ & $D^{*} \bar{D}^{*}$ & $4013_{-11}^{+4}(\mathrm{~V})$ & $3983_{-32}^{+17}(\mathrm{~V})$ & $?$ \\
\hline $1\left(1^{+}\right)$ & $D^{*} B^{*}$ & $7333.6_{-4.2}^{\dagger}(\mathrm{V})$ & $7328_{-14}^{+5}(\mathrm{~V})$ & $?$ \\
\hline
\end{tabular}

Two combinations of the LEC's can be obtained from the properties of the $X(3872)$ resonance ${ }^{4}$, assuming that it is a $\left(D \bar{D}^{*}-D^{*} \bar{D}\right) / \sqrt{2}$ bound state. The isospin properties of the $X(3872)$ molecule are mainly determined by its mass, which is only few tens of keV below the $D^{0} \bar{D}^{* 0}$ threshold, making relevant the around $8 \mathrm{MeV}$ difference between the threshold of the neutral and of the charged $\left(D^{+} D^{*-}\right)$ channels 7 , 14].

Assuming HFS, a third independent combination of LEC's is fixed from the isovector $Z_{b}(10610)$ resonance, described as $\left(B \bar{B}^{*}+B^{*} \bar{B}\right) / \sqrt{2}$ molecular state. Note, HQSS predicts the interaction of the $B^{*} \bar{B}^{*}$ system with $I=1, J^{P C}=$ $1^{+-}$quantum numbers to be identical to that of the $B \bar{B}^{*}$ pair in the $Z_{b}(10610)$ sector. Thus, HQSS naturally explains the approximate degeneracy of the $Z_{b}(10610)$ and $Z_{b}(10650)$ resonances [4, 5].

There are various $D^{(*)} \bar{D}^{(*)}, B^{(*)} \bar{B}^{(*)}$ and $D^{(*)} \bar{B}^{(*)}$ sectors where the interaction is completely fixed by the the three linear combinations of LEC's obtained from the $X(3872)$ and the $Z_{b}(10610)$ resonances, which allows us to make predictions on the existence of additional molecular states, by solving the LSE as previously commented. Some of these approximate predictions from [15] are collected in Table []

\section{TRIPLY HEAVY PENTAQUARKS}

The existence of heavy meson-antimeson molecules implies the possibility of partners composed of a heavy meson and a doubly-heavy baryon (triply-heavy pentaquarks) 24]. This is based on the approximate heavy antiquarkdiquark symmetry (HADS) that emerges from the observation that the interactions of heavy color triplet objects with the light degrees of freedom (quarks and gluons) are independent of the heavy color triplet's spin and mass [25].

A first consequence is that the spectrum of baryons with two heavy quarks can be related to the spectrum of heavy light mesons with the same light degree of freedom quantum numbers [25]. The heavy diquark component of the baryon forms a color anti-triplet with a characteristic length scale of $1 /\left(m_{Q} v\right)$, where $m_{Q}$ is the mass of the heavy quarks and $v$ their velocity. The length scale of the diquark is smaller than the typical QCD length scale $1 / \Lambda_{\mathrm{QCD}}$ and hence we can treat the diquark as point-like if the quarks are heavy enough.

Within this scheme, we hint the existence of several baryonic partners of the $X(3872)$ with isospin $I=0$ and $J^{P}=\frac{5}{2}^{-}$or $\frac{3}{2}^{-}$. Moreover, we predict various $\Xi_{b b}^{*} \bar{B}^{*}$ triply-heavy pentaquarks with quantum numbers $I\left(J^{P}\right)=1\left(\frac{1}{2}^{-}\right)$ and $I\left(J^{P}\right)=1\left(\frac{3}{2}^{-}\right)$partners of the $Z_{b}(10610) B^{*} \bar{B}^{*}$ molecule.

\footnotetext{
${ }^{4}$ Mass and the isospin violating ratio of the decay amplitudes for the $X(3872) \rightarrow J / \psi \pi \pi$ and $X(3872) \rightarrow J / \psi \pi \pi \pi$ transitions, $R_{X(3872)}=$ $0.26 \pm 0.07$ deduced in [18] using the experimental ratio $\mathcal{B}_{X}=\Gamma[X \rightarrow J / \Psi \rho] / \Gamma[X \rightarrow J / \Psi \omega]=1.3 \pm 0.5[19]$.
} 
TABLE II: Doubly-heavy baryon-heavy meson molecules masses. The error in the masses of the isoscalar states is a consequence of the approximate nature of HADS. For the isovector states, different error sources have been taken into account: the uncertainty in the $Z_{b}$ binding, in the isospin breaking decays of the $X$ and in the HADS breaking. For simplicity, we only show an unique error obtained by adding in quadratures all the previous ones. $M_{\text {th }}$ represents the threshold, and all masses are given in units of $\mathrm{MeV}$. When we decrease the strength of the potential to account for the various uncertainties, in some cases (marked with $\dagger$ in the table) the bound state pole reaches the threshold and the state becomes virtual. The cases with a virtual state pole at the central value are marked by $[\mathrm{V}]$, for which $\dagger \dagger$ means that the pole evolves into a bound state one and N/A means that the pole is far from the threshold with a momentum larger than $1 \mathrm{GeV}$ so that it is both undetectable and beyond the EFT range. For further details see [24].

\begin{tabular}{lccccc} 
State & $I\left(J^{P}\right)$ & $V^{\mathrm{LO}}$ & Thresholds & $\mathrm{M}(\Lambda=0.5 \mathrm{GeV})$ & $\mathrm{M}(\Lambda=1 \mathrm{GeV})$ \\
\hline$\Xi_{c c}^{*} D^{*}$ & $0\left(\frac{5}{2}^{-}\right)$ & $C_{0 a}+C_{0 b}$ & 5715 & $\left(M_{\mathrm{th}}-10\right)_{-15}^{+10}$ & $\left(M_{\mathrm{th}}-19\right)_{-44}^{\dagger}$ \\
$\Xi_{c c}^{*} \bar{B}^{*}$ & $0\left(\frac{5}{2}^{-}\right)$ & $C_{0 a}+C_{0 b}$ & 9031 & $\left(M_{\mathrm{th}}-21\right)_{-19}^{+16}$ & $\left(M_{\mathrm{th}}-53\right)_{-59}^{+45}$ \\
$\Xi_{b b}^{*} D^{*}$ & $0\left(\frac{5}{2}^{-}\right)$ & $C_{0 a}+C_{0 b}$ & 12160 & $\left(M_{\mathrm{th}}-15\right)_{-11}^{+9}$ & $\left(M_{\mathrm{th}}-35\right)_{-31}^{+25}$ \\
$\Xi_{b b}^{*} \bar{B}^{*}$ & $0\left(\frac{5}{2}^{-}\right)$ & $C_{0 a}+C_{0 b}$ & 15476 & $\left(M_{\mathrm{th}}-29\right)_{-13}^{+12}$ & $\left(M_{\mathrm{th}}-83\right)_{-40}^{+33}$ \\
$\Xi_{b c}^{\prime} D^{*}$ & $0\left(\frac{3}{2}^{-}\right)$ & $C_{0 a}+C_{0 b}$ & 8967 & $\left(M_{\mathrm{th}}-14\right)_{-13}^{+11}$ & $\left(M_{\mathrm{th}}-30\right)_{-40}^{+27}$ \\
$\Xi_{b c}^{\prime} \bar{B}^{*}$ & $0\left(\frac{3}{2}^{-}\right)$ & $C_{0 a}+C_{0 b}$ & 12283 & $\left(M_{\mathrm{th}}-27\right)_{-16}^{+15}$ & $\left(M_{\mathrm{th}}-74\right)_{-51}^{+445}$ \\
$\Xi_{b c}^{*} D^{*}$ & $0\left(\frac{5}{2}^{-}\right)$ & $C_{0 a}+C_{0 b}$ & 9005 & $\left(M_{\mathrm{th}}-14\right)_{-13}^{+11}$ & $\left(M_{\mathrm{th}}-30\right)_{-40}^{+27}$ \\
$\Xi_{b c}^{*} \bar{B}^{*}$ & $0\left(\frac{5}{2}^{-}\right)$ & $C_{0 a}+C_{0 b}$ & 12321 & $\left(M_{\mathrm{th}}-27\right)_{-16}^{+15}$ & $\left(M_{\mathrm{th}}-74\right)_{-51}^{+46}$ \\
\hline$\Xi_{b b} \bar{B}^{+1}$ & $1\left(\frac{1}{2}^{-}\right)$ & $C_{1 a}$ & 15406 & $\left(M_{\mathrm{th}}-0.3\right)_{-2.5}^{\dagger}$ & $\left(M_{\mathrm{th}}-12\right)_{-15}^{+11}$ \\
$\Xi_{b b} \bar{B}^{*}$ & $1\left(\frac{1}{2}^{-}\right)$ & $C_{1 a}+\frac{2}{3} C_{1 b}$ & 15452 & $\left(M_{\mathrm{th}}-0.9\right)[\mathrm{V}]_{\dagger \dagger}^{\mathrm{N} / \mathrm{A}}$ & $\left(M_{\mathrm{th}}-16\right)_{-17}^{+14}$ \\
$\Xi_{b b} \bar{B}^{*}$ & $1\left(\frac{3}{2}^{-}\right)$ & $C_{1 a}-\frac{1}{3} C_{1 b}$ & 15452 & $\left(M_{\mathrm{th}}-1.2\right)_{-2.9}^{+}$ & $\left(M_{\mathrm{th}}-10\right)_{-13}^{+9}$ \\
$\Xi_{b b}^{*} \bar{B}^{+1}$ & $1\left(\frac{3}{2}^{-}\right)$ & $C_{1 a}$ & 15430 & $\left(M_{\mathrm{th}}-0.3\right)_{-2.4}^{\dagger}$ & $\left(M_{\mathrm{th}}-12\right)_{-13}^{+11}$ \\
$\Xi_{b b}^{*} \bar{B}^{*}$ & $1\left(\frac{1}{2}^{-}\right)$ & $C_{1 a}-\frac{5}{3} C_{1 b}$ & 15476 & $\left(M_{\mathrm{th}}-8\right)_{-7}^{+8}$ & $\left(M_{\mathrm{th}}-5\right)_{-8}^{\dagger}$ \\
$\Xi_{b b}^{*} \bar{B}^{*}$ & $1\left(\frac{3}{2}^{-}\right)$ & $C_{1 a}-\frac{2}{3} C_{1 b}$ & 15476 & $\left(M_{\mathrm{th}}-2.5\right)_{-3.6}^{\dagger}$ & $\left(M_{\mathrm{th}}-9\right)_{-11}^{+9}$ \\
$\Xi_{b b}^{*} \bar{B}^{*}$ & $1\left(\frac{5}{2}^{-}\right)$ & $C_{1 a}+C_{1 b}$ & 15476 & $\left(M_{\mathrm{th}}-4.3\right)[\mathrm{V}]_{+3.3}^{\mathrm{N} / \mathrm{A}}$ & $\left(M_{\mathrm{th}}-18\right)_{-19}^{+117}$
\end{tabular}

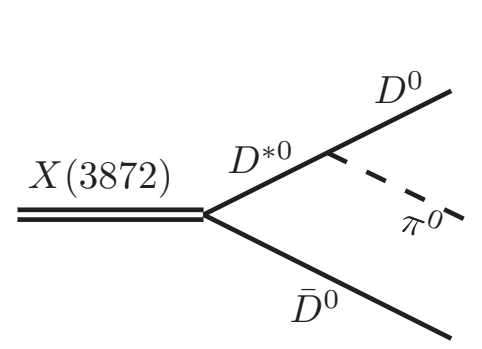

(a)

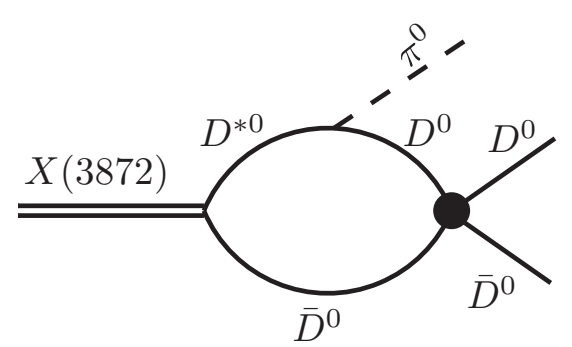

(b)

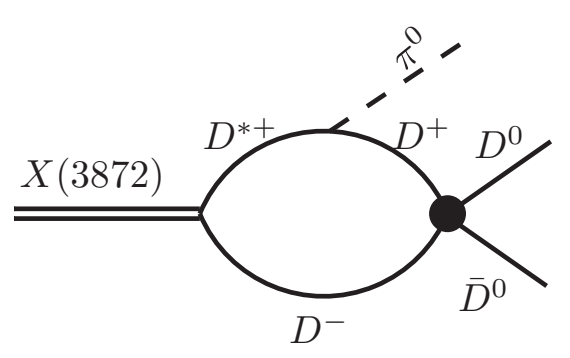

(c)

FIG. 1: Feynman diagrams for the decay $X(3872) \rightarrow D^{0} \bar{D}^{0} \pi^{0}$. The charge conjugate channel is not shown but included in the calculations.

We compile some of these approximate predictions from [15] in Table I] They are subject to larger uncertainties than those collected in Table \ since violations of HADS are expected to be larger than HQSS and HFS ones. i.e., $\mathcal{O}\left(\Lambda_{\mathrm{QCD}} /\left(m_{Q} v\right)\right)$ vs $\mathcal{O}\left(\Lambda_{\mathrm{QCD}} / m_{Q}\right)$.

\section{THE $X(3872) \rightarrow D^{0} \bar{D}^{0} \pi^{0}$ DECAY AND THE LONG-DISTANCE STRUCTURE OF THE $X(3872)$ RESONANCE}

Within the molecular picture of the $X(3872)$ resonance, in its decay modes with a charmonium in the final state $\left(J / \psi \pi \pi, J / \psi 3 \pi, J / \psi \gamma\right.$ and $\left.\psi^{\prime} \gamma\right)$, the heavy quarks of the $D \bar{D}^{*}$ meson pair have to recombine to form the final charmonium. As a consequence, these processes are not sensitive to the $D \bar{D}^{*}$ wave function at long distances which is governed by the binding energy, but rather they are determined by the short distance part of the $X(3872)$ wavefunction [7].

The transition from the charm-anti-charm meson pair into the $J / \psi$ plus pions (or a photon), occurs at a distance much smaller than both the size of the $X(3872)$ as a hadronic molecule and the range of forces between the $D$ and 
$\bar{D}^{*}$ mesons. However, in the case of the $X(3872) \rightarrow D^{0} \bar{D}^{0} \pi^{0}$ decay, one of the constituent hadrons $\left(D^{0}\right)$ is in the final state and the rest of the final particles are products of the decay of the other constituent hadron $\left(\bar{D}^{* 0}\right)$ of the $X(3872)$ molecule. Thus, in this decay the relative distance between the $D \bar{D}^{*}$ mesons can be as large as allowed by the size of the $X(3872)$ resonance, since the final state is produced by the decay of the $\bar{D}^{*}$ meson instead of a rescattering transition. Actually, it can be proved that within some approximations, the $d \Gamma / d\left|\vec{p}_{D^{0}}\right|$ distribution is related to the $X(3872)$ wave-function $\Psi\left(\vec{p}_{D^{0}}\right)[26]$.

We have estimated the $X(3872) \rightarrow D^{0} \bar{D}^{0} \pi^{0}$ decay width by evaluating the diagrams depicted in Fig. 1 . The tree level contribution is fully determined by the $D^{0} \bar{D}^{* 0} \pi$ coupling $\left(g / f_{\pi}\right)$, the $X(3872)$ mass and its coupling constant to the neutral $D^{0} \bar{D}^{* 0}$ channel $\left(g_{0}^{X}\right)$, which is determined by the residue of the $T$-matrix element at the $X(3872)$ pole. We find [26]

$$
T_{\text {tree }}=-2 i \frac{g g_{0}^{X}}{f_{\pi}} \sqrt{M_{X}} M_{D^{* 0}} M_{D^{0} \vec{\epsilon}_{X}} \cdot \vec{p}_{\pi}\left(\frac{1}{p_{12}^{2}-M_{D^{* 0}}^{2}}+\frac{1}{p_{13}^{2}-M_{D^{* 0}}^{2}}\right),
$$

where $\vec{\epsilon}_{X}$ is the polarization vector of the $X(3872), \vec{p}_{\pi}$ is the three-momentum of the pion, $p_{12}$ and $p_{13}$ are the four momenta of the $\pi^{0} D^{0}$ and $\pi^{0} \bar{D}^{0}$ systems, respectively. Taking into account the phase space,

$$
d \Gamma=\frac{1}{(2 \pi)^{3}} \frac{1}{32 M_{X}^{3}} \overline{|T|}^{2} d m_{12}^{2} d m_{23}^{2}
$$

with the invariant masses $m_{12}^{2}=p_{12}^{2}$ and $m_{23}^{2}=\left(M_{X}^{2}+m_{\pi^{0}}^{2}+2 M_{D^{0}}^{2}-m_{12}^{2}-p_{13}^{2}\right)$ of the final $\pi^{0} D^{0}$ and $D^{0} \bar{D}^{0}$ pairs, we readily obtain

$$
\begin{aligned}
\Gamma_{\text {tree }} & =\frac{g^{2}}{192 \pi^{3} f_{\pi}^{2}}\left(g_{0}^{X} \frac{M_{D^{0}} M_{D^{* 0}}}{M_{X}}\right)^{2} \int_{\left(M_{D^{0}}+m_{\pi}^{0}\right)^{2}}^{\left(M_{X}-M_{D^{0}}\right)^{2}} d m_{12}^{2} \\
& \times \int_{\left(m_{23}^{2}\right)_{(\min )}}^{\left(m_{23}^{2}\right)_{(\max )}} d m_{23}^{2}\left(\frac{1}{p_{12}^{2}-M_{D^{* 0}}^{2}}+\frac{1}{p_{13}^{2}-M_{D^{* 0}}^{2}}\right)^{2}\left|\vec{p}_{\pi}\right|^{2}
\end{aligned}
$$

where $\left|\vec{p}_{\pi}\right|=\lambda^{1 / 2}\left(M_{X}^{2}, m_{23}^{2}, m_{\pi^{0}}^{2}\right) / 2 M_{X}$ is the pion momentum in the $X(3872)$ center of mass frame $[\lambda(x, y, z)=$ $\left.x^{2}+y^{2}+z^{2}-2(x y+y z+x z)\right]$. In addition, for a given value of $m_{12}^{2}$, the range of $m_{23}^{2}$ is determined by:

$$
\left(m_{23}^{2}\right)_{(\max , \min )}=\left(E_{D}^{*}+E_{\bar{D}}^{*}\right)^{2}-\left(p_{D}^{*} \mp p_{\bar{D}}^{*}\right)^{2}
$$

with $E_{D}^{*}=\left(m_{12}^{2}-m_{\pi^{0}}^{2}+M_{D^{0}}^{2}\right) / 2 m_{12}$ and $E_{\bar{D}}^{*}=\left(M_{X}^{2}-m_{12}^{2}-M_{D^{0}}^{2}\right) / 2 m_{12}$ the energies of the $D^{0}$ and $\bar{D}^{0}$ in the $m_{12}$ rest frame, respectively, and $p_{D, \bar{D}}^{*}$ the moduli of their corresponding three momenta. In Ref. [26], we found

$$
\Gamma\left(X(3872) \rightarrow D^{0} \bar{D}^{0} \pi^{0}\right)_{\text {tree }}=44.0_{-7.2}^{+2.4}\left(42.0_{-7.3}^{+3.6}\right) \mathrm{keV},
$$

where the values outside and inside the parentheses are obtained with UV Gaussian cutoffs of $\Lambda=0.5$ and 1 GeV, respectively, and the errors (grey bands in Fig. 2) reflect the uncertainty in the inputs $\left(M_{X(3872)}\right.$ and the ratio of decay amplitudes for the $X(3872) \rightarrow J / \psi \rho$ and $X(3872) \rightarrow J / \psi \omega$ decays $)$.

Since we published these results, new high precision measurements of the masses of the $D^{0}$ and $D^{* 0}$ mesons have become available [27, 28], which have led to a more precise determination of the $X(3872)$ binding energy, $B=13 \pm 192$ $\mathrm{keV}$ [28]. To obtain the central values and the errors of Eq. (5) and the $68 \%$ confident level (CL) bands displayed in Fig. 2, we used $B=160 \pm 170 \mathrm{keV}$, and a Monte Carlo simulation was performed to propagate errors ${ }^{5}$. In the simulation, we rejected $X(3872)$ binding energies values smaller than $10 \mathrm{keV}$, and those values were set to this minimum value. This effectively amounts to consider $B=160_{-150}^{+170} \mathrm{keV}$, since the Gaussian distribution of binding energies was truncated. We slightly decreased the lower error to guaranty a bound state with a CL larger than $68 \%$, since the scheme followed in [26] only allows the computation of the width when the $X(3872)$ state is bound. This binding energy range leads to $g_{0}^{X}=0.35_{-0.18}^{+0.08}\left(0.34_{-0.18}^{+0.07}\right) \mathrm{GeV}^{-1 / 2}$ (the two values correspond to the $\Lambda=0.5$ and $1 \mathrm{GeV}$ choices of the UV regulator), which is not compatible with zero ${ }^{6}$. Thus, the lower grey bands in Fig. 1 turn out not to be compatible with a zero width either. However, the new determination of $B=13 \pm 192 \mathrm{keV}$ makes

\footnotetext{
5 The error on the threshold energy $\left(M_{D^{0}}+M_{D^{* 0}}\right), \sim 120 \mathrm{keV}$, was not taken into account in Ref. [26].

${ }^{6}$ In [26], larger lower errors for the coupling $g_{0}^{X}=0.35_{-0.29}^{+0.08}\left(0.34_{-0.29}^{+0.07}\right) \mathrm{GeV}^{-1 / 2}$ were quoted. These correspond to a minimum of the binding energy $(\sim 0.1 \mathrm{keV})$ much closer to zero, though in the calculation of the width, the $10 \mathrm{keV}$ cut, mentioned above, was used.
} 

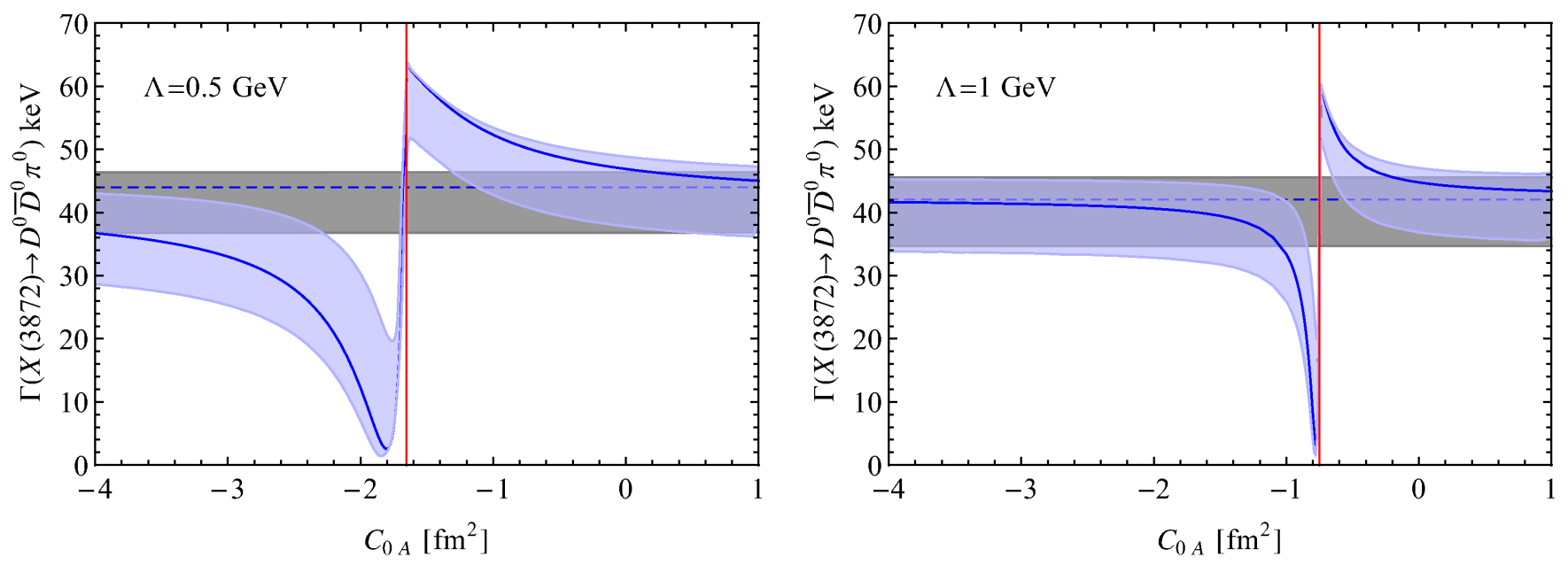

FIG. 2: $X(3872) \rightarrow D^{0} \bar{D}^{0} \pi^{0}$ partial decay width as a function of $C_{0 A}$. The UV cutoff is set to $\Lambda=0.5 \mathrm{GeV}(1 \mathrm{GeV})$ in the left (right) panel. The blue error bands contain $D \bar{D}$ FSI effects, while the grey bands stand for the tree level predictions (see Ref [26] for details).

much more probable the very low binding energies close to zero, or an unbound resonance. In this case, we would like to point out that the decay width should decrease, and eventually should vanish, as the binding energy approaches zero. This is because for very small binding energies, all the couplings of a bound state tend to zero when the mass of the bound state gets closer to the lowest threshold [29]. For the case of the $X(3872)$, this was re-derived in [7] and explicitly shown that both the neutral $X(3872) D^{0} \bar{D}^{* 0}\left(g_{0}^{X}\right)$ and charged $X(3872) D^{+} \bar{D}^{*-}$ couplings scale as $B^{\frac{1}{4}}$. Because of the quite limited phase space available in this $p$-wave decay, the decay width, however, increases very rapidly as the binding energy departs from zero. In any case, the lower errors displayed in Eq. (5) and Fig. 2] should be now considered with some caution. Moreover, the effect of the $D^{* 0}$ width, neglected in the present calculation, becomes sizable for binding energies below $10 \mathrm{keV}$.

The last two diagrams in Fig. 1 account for the $D \bar{D} \rightarrow D \bar{D}$ final state interaction (FSI) effects, which are considered by means of the appropriated linear combinations of the isoscalar and isovector $T$-matrices. To obtain these scattering amplitudes, the LO contact potential, involving the four LEC's $C_{0 A}, C_{0 B}, C_{1 A}$ and $C_{1 B}$, is used [14, 26]. As commented, the $X(3872)$ and $Z_{b}(10610)$ inputs determine only three of the four counter-terms. The value of $C_{0 A}$ is not fixed, and thus the $D \bar{D}$ FSI effects on this decay are not fully determined. As can be seen in Fig. 2 these effects might be quite large, because for a certain range of $C_{0 A}$ values, a near-threshold isoscalar $D \bar{D}$ bound state could be dynamically generated [13, 14]. If in future experiments the partial decay width is measured, a significant deviation from the predicted tree level value will indicate a FSI effect, which might be used to extract the value of $C_{0 A}$. However as discussed above, this could be obscured if turned out that the actual binding energy of the $X(3872)$ state is smaller than let us say $10 \mathrm{keV}$.

\section{Acknowledgments}

C. H.-D. thanks the support of the JAE-CSIC Program. This work is supported in part by the DFG and the NSFC through funds provided to the Sino-German CRC 110 "Symmetries and the Emergence of Structure in QCD", by the NSFC (Grant No. 11165005), by the Spanish Ministerio de Economía y Competitividad and European FEDER funds under the contract FIS2011-28853-C02-02 and the Spanish Consolider-Ingenio 2010 Programme CPAN (CSD200700042), by Generalitat Valenciana under contract PROMETEOII/2014/0068 and by the EU HadronPhysics3 project, grant agreement no. 283286.

[1] Voloshin, M. B., Okun, L. B.: JETP Lett. 23, 333 (1976)

[2] De Rujula, A., Georgi, H. and Glashow, S. L.: Phys. Rev. Lett. 38, 317 (1977)

[3] Choi, S. K., et al.: Phys. Rev. Lett. 91, 262001 (2003)

[4] Bondar, A., et al.: Phys. Rev. Lett. 108, 122001 (2012) 
[5] Adachi, I., et al.: Evidence for a $Z_{b}^{0}(10610)$ in Dalitz analysis of $\Upsilon(5 S) \rightarrow \Upsilon(n S) \pi^{0} \pi^{0}$ Preprint arXiv:1207.4345 (2012)

[6] Aaij, R., et al.: Phys. Rev. Lett. 110, 222001 (2013)

[7] Gamermann, D., Nieves, J., Oset, E., Ruiz Arriola, E.. Phys. Rev. D 81, 014029 (2010)

[8] Guo, F. K., Hanhart, C., Meißner, U.G.: Phys. Rev. Lett. 102, 242004 (2009)

[9] Bondar, A. E., Garmash, A., Milstein, A. I., Mizuk, R., Voloshin, M. B.: Phys. Rev. D 84, 054010 (2011)

[10] Voloshin, M. B.: Phys. Rev. D 84, 031502 (2011)

[11] Mehen, T., Powell, J. W.: Phys. Rev. D 84, 114013 (2011)

[12] Nieves, J., Valderrama, M. P.: Phys. Rev. D 84, 056015 (2011)

[13] Nieves, J., Valderrama, M. P.: Phys. Rev. D 86, 056004 (2012)

[14] Hidalgo-Duque, C., Nieves, J., Valderrama, M. P.: Phys. Rev. D 87, 076006 (2013)

[15] Guo, F. K., Hidalgo-Duque, C., Nieves, J., Valderrama, M. P.: Phys. Rev. D 88, 054007 (2013)

[16] AlFiky, M. T., Gabbiani, F., Petrov, A. A.: Phys. Lett. B 640, 238 (2006)

[17] Valderrama,M.P.: Phys. Rev. D 85, 114037 (2012)

[18] Hanhart,C., Kalashnikova, Y.S., Kudryavtsev, A.E., Nefediev, A. V.: Phys. Rev. D 85, 011501 (2012)

[19] Choi, S. K., et al.: Phys. Rev. D 84, 052004 (2011)

[20] Beringer, J., et al.: Phys. Rev. D 86, 010001 (2012)

[21] Adachi. I., et al.: Study of Three-Body $\Upsilon$ (10860) Decays Preprint arXiv:1209.6450 (2012)

[22] Ablikim, M., et al: Phys. Rev. Lett. 110, 252001 (2013)

[23] Liu, Z., et al.: Phys. Rev. Lett. 110, 252002 (2013)

[24] Guo, F. K., Hidalgo-Duque, C., Nieves, J., Valderrama, M. P.: Phys. Rev. D 88, 0540142013.

[25] Savage, M. J., Wise, M. B.: Phys. Lett. B 248, 177 (1990).

[26] Guo, F. K., Hidalgo-Duque, C., Nieves, J., Ozpineci, A., Valderrama, M. P.: Eur. Phys. J. C 74, 2885 (2014)

[27] Tomaradze, A., Dobbs, S., Xiao, T., Seth, K. K., Bonvicini, G.: Phys. Rev. D 89, 031501 (2014)

[28] Seth, K.K.: New high precision measurements of the masses $M\left(D^{0}\right)$ and $M\left(D^{* 0}\right)$ and the binding energy of $X(3872)$, talk at Quarkonium 2014 Workshop at CERN, November 2014.

[29] Weinberg, S.: Phys. Rev. 137, B672 (1965). 